\title{
Laboratory Evaluation of Placement Behavior of Microgels for Conformance Control in Reservoirs Containing Superpermeable Channels
}

\author{
Yang Zhao ${ }^{a}$, Baojun Baia ${ }^{*}$ \\ ${ }^{a}$ Department of Geosciences and Geological and Petroleum Engineering, Missouri University of Science and \\ Technology, 1400 N Bishop Ave, Rolla, MO, 65409, United States \\ *baib@mst.edu
}

\section{SUPPORTING INFORMATION}

The injection pressure, pressure gradient, and resistance factor during gel injection in the experiments (except for Exp S4) are presented in Figures S1-S6.
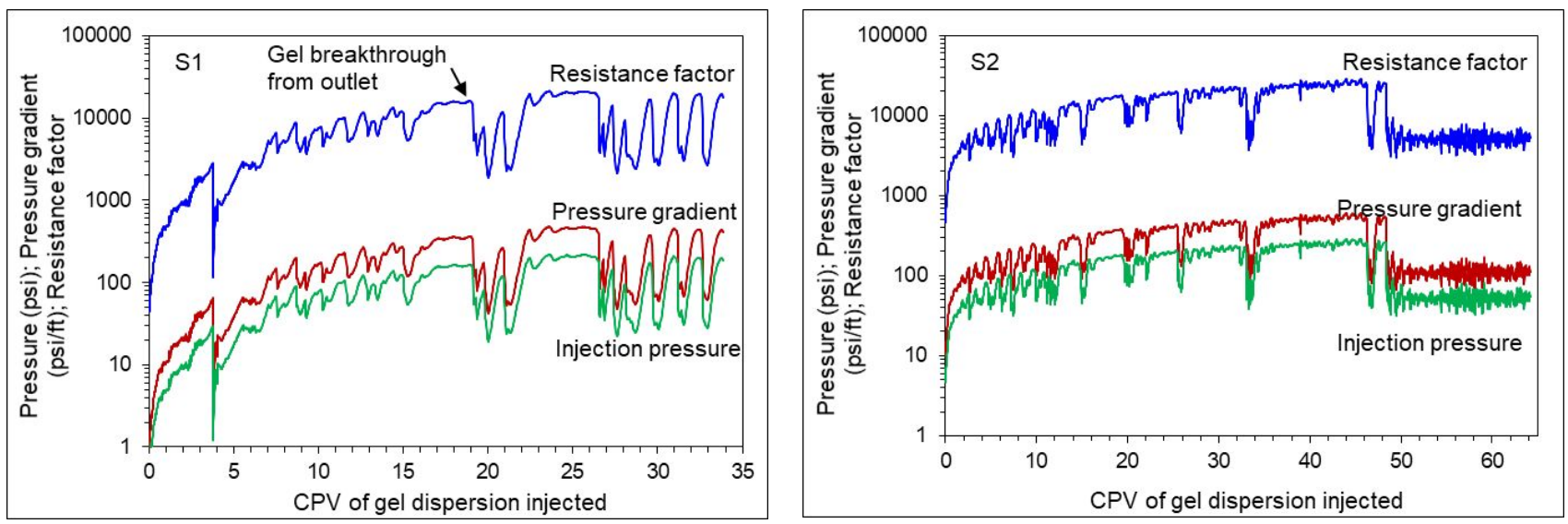

Figure S1. Injection pressure, pressure gradient and Figure S2. Injection pressure, pressure gradient and resistance factor during gel injection $(\operatorname{Exp} \mathrm{S1})$. resistance factor during gel injection (Exp S2).
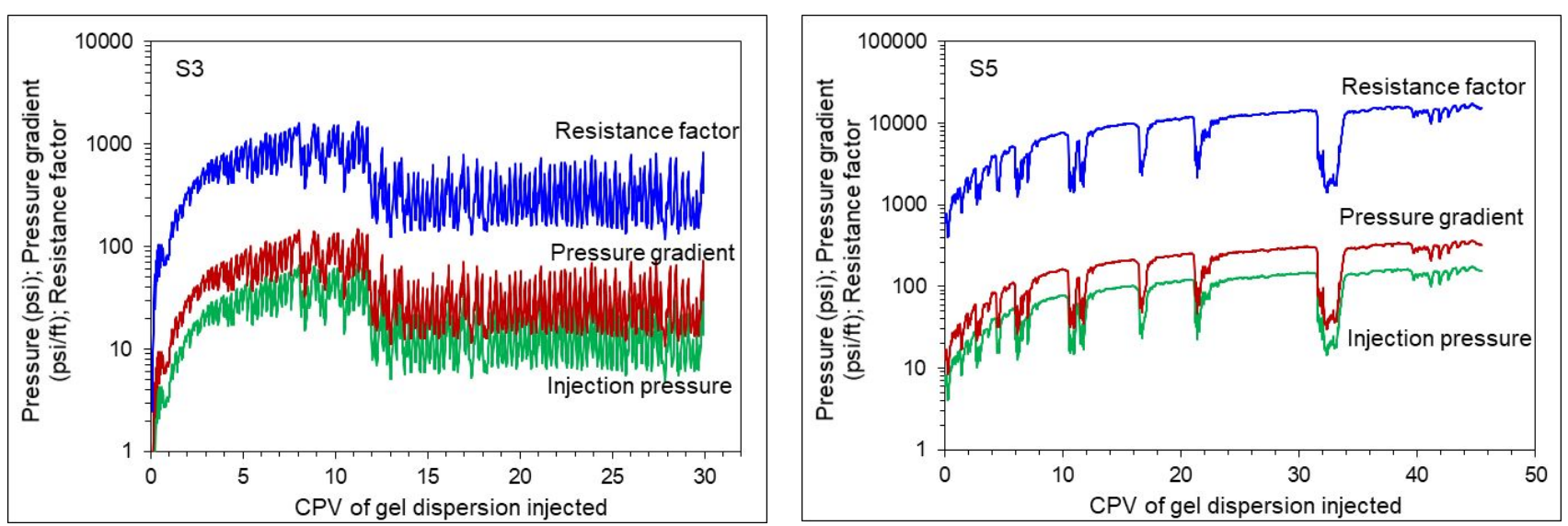

Figure S3. Injection pressure, pressure gradient and Figure S4. Injection pressure, pressure gradient and resistance factor during gel injection (Exp S3).

resistance factor during gel injection (Exp S5). 

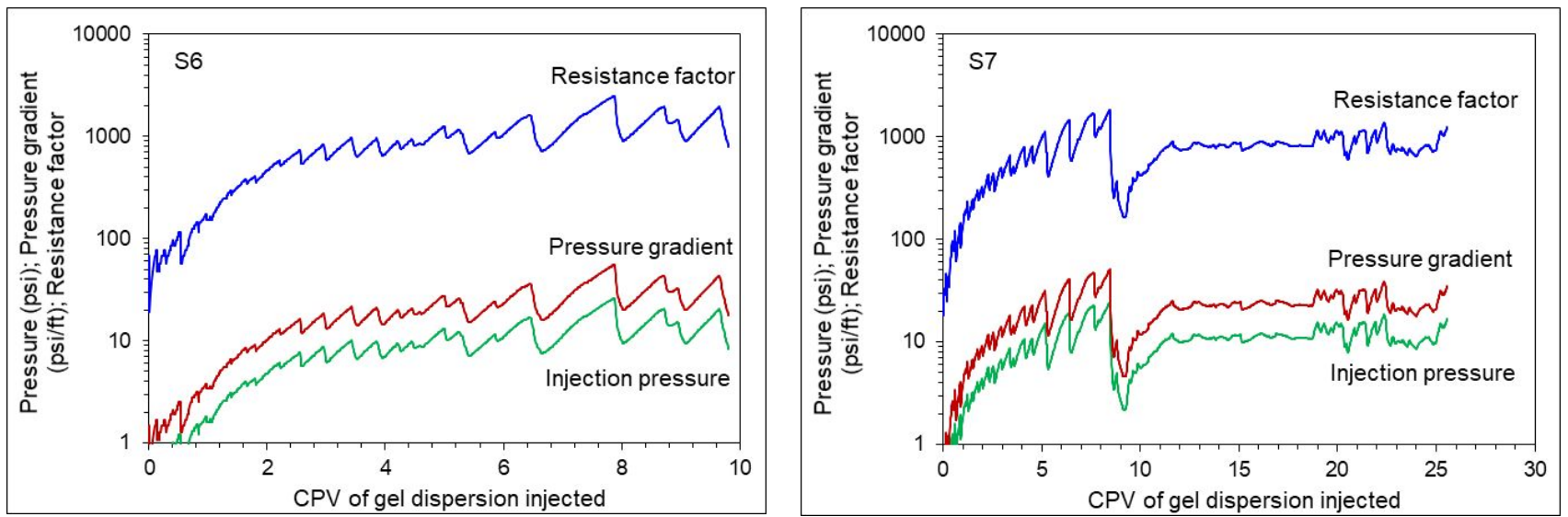

Figure S5. Injection pressure, pressure gradient and Figure S6. Injection pressure, pressure gradient and resistance factor during gel injection (Exp S6). resistance factor during gel injection (Exp S7). 